\title{
Corrigendum: A playroom as novel swine enrichment
}

Blair Casey, BS, ALAT, Dawn Abney, BA, LAT \& Evelyn Skoumbourdis, MS, LATg Lab Anim. (NY) 36 (3), 32-34 (2007).

The correct spelling of the third author's name is Evelyn Skoumbourdis. 